\title{
Interlaboratory evaluation of endotoxin analyses in agricultural dusts-comparison of LAL assay and mass spectrometry
}

\author{
Stephen J. Reynolds, ${ }^{* a}$ Donald K. Milton, ${ }^{b}$ Dick Heederik, ${ }^{c}$ Peter S. Thorne, ${ }^{d}$ Kelley J. \\ Donham, ${ }^{e}$ Elizabeth A. Croteau, ${ }^{d}$ Kevin M. Kelly, ${ }^{d}$ Jeroen Douwes, ${ }^{c}$ Daniel Lewis, ${ }^{e}$ \\ Mike Whitmer, ${ }^{e}$ Ian Connaughton, ${ }^{f}$ Sharon Koch, ${ }^{f}$ Per Malmberg, ${ }^{g}$ Britt-Marie Larsson, ${ }^{g}$ \\ Jim Deddens, ${ }^{h}$ Anita Saraf ${ }^{i}$ and Lennart Larsson ${ }^{i}$
}

\author{
${ }^{a}$ Department of Environmental and Radiological Health Sciences, Colorado State University, \\ Ft.Collins, CO 80523-1681,USA.E-mail: Stephen.Reynolds@Colostate.edu;

Received 30th June 2005, Accepted 13th September 2005

First published as an Advance Article on the web 11th October 2005

Endotoxin exposure is associated with wheeze and asthma morbidity, while early life exposure may reduce risk of allergy and asthma. Unfortunately, it is difficult to compare endotoxin results from different laboratories and environments. We undertook this study to determine if lipopolysaccharide (LPS) extraction efficiency could account for differences among laboratories. We generated and collected aerosols from chicken and swine barns, and corn processing. We randomly allocated side-by-side filter samples to five laboratories for Limulus assay of endotoxin. Lyophilized aliquots of filter extracts were analyzed for 3-hydroxy fatty acids (3-OHFAs) as a marker of LPS using gas chromatography-mass spectrometry. There were significant differences in endotoxin assay and GC-MS (LPS) results between laboratories for all dust types $(p<0.01)$. Patterns of differences between labs varied by dust type. Relationships between assay and GC/MS results also depended on dust type. The percentages of individual 3-OHFA chain lengths varied across labs $(p<0.0001)$ suggesting that each lab recovered a different fraction of the LPS available. The presence of large amounts of particle associated LPS and absence of a freezing thawing cycle were associated with lower correlations between LPS and bioactivity, consistent with an absence of Limulus response to cellbound endotoxin. These data suggest that extraction methods affect endotoxin measurements. The LAL methods may be most suitable when comparing exposures within similar environments; GC-MS offers additional information helpful in optimizing sample treatment and extraction. GC-MS may be of use when comparing across heterogeneous environments and should be considered for inclusion in future studies of human health outcomes.

\section{Introduction}

Lipopolysaccharides (LPS), components of the outer membrane of gram-negative bacteria that are the essential chemical moiety of gram-negative bacterial endotoxin, are associated with respiratory symptoms and decrements in pulmonary function in a variety of agricultural and industrial environments. ${ }^{1-9}$ Low level endotoxin exposure has recently been associated with non-specific building-related symptoms among office workers ${ }^{10,11}$ with increased asthma severity, ${ }^{12,13}$ and with repeated wheeze in infancy. ${ }^{14,15}$ However, endotoxin exposure early in life may be a protective factor that can reduce risk of childhood asthma and atopy. ${ }^{16-18}$
Progress has been made in establishing dose-response relationships for endotoxin and respiratory disease ${ }^{4,5,8,15,19}$ however, there are some older reports in the literature of very high exposures without apparent symptoms, ${ }^{20}$ and of very low exposures with a possible dose-response relationship, ${ }^{21}$ where endotoxin may have merely correlated with another causative agent. Occasional studies have also reported very high ${ }^{22}$ or very low ${ }^{10}$ endotoxin exposures in office buildings compared with reports from other labs. ${ }^{11,23}$ In general, the agreement between studies on the thresholds for respiratory effects is within an order of magnitude. But due partly to lack of standard protocols and reagents there has been a lack of agreement, especially in some of the earlier studies, which 
made promogulation of exposure standards until recently controversial. ${ }^{24,25}$ Proposals for standard protocols have recently been published, but these are not completely validated and not yet widely applied.

Most researchers have used the Limulus amebocyte lysate (LAL) assay to measure endotoxin exposures, and the response of this assay depends on the relative reactivity with Limulus lysate of endotoxins from different bacteria in various aerosol matrices. ${ }^{26-28}$ Variability in laboratory methods for collection of samples (filter types), sample handling and storage (use of desiccant, storage temperature), analysis of samples (extraction media, extraction time, rocking, sonication, temperature, assay type, control standards, and LAL lots), and variation in the reporting of results (units) have also contributed to the difficulty of identifying universal exposure-response thresholds. ${ }^{29-35}$

To resolve this problem, several interlaboratory comparisons have now been conducted using cotton, poultry, swine, and corn dusts. ${ }^{32,35-37}$ These studies have demonstrated that differences between laboratories are not necessarily consistent and depend on the environmental source of the endotoxin. ${ }^{32,35}$ We recently compared six laboratories using LAL-based assays for analysis of organic dusts from three agricultural environments. ${ }^{37}$ In contrast to previous comparisons, interlot variation in LAL could not have contributed to differences in this study. Precision of assays performed within labs was good, however, we observed significant differences between endotoxin estimates from laboratories for all three dust types $(p<$ 0.01 ). The pattern of differences between labs varied by dust type and may be related to the extraction and analytical methods.

In contrast to biological assay methods, chemical analyses using gas chromatography-mass spectrometry (GC-MS) have focused on quantification of 3-hydroxy fatty acids (3-OHFA) in LPS. ${ }^{7,28,38-42}$ This technique has been applied to study dusts from poultry, swine, fiberglass manufacturing, and household environments. The correlations between bioassay using LAL and 3-OHFA concentrations in dust have been variable. $7,28,38,40,42$

The goal of this study was to use GC-MS to evaluate the contribution of extraction procedures to differences in endotoxin measurements between labs using Limulus-based assays. We report here the results from five laboratories that analyzed experimental organic dust aerosols from three agricultural environments.

\section{Experimental methods}

Aerosol generation and LAL endotoxin assay methods were described previously ${ }^{37}$ and are summarized briefly.

\section{Aerosol generation and sample collection}

Bulk dust collected from a chicken laying facility, a swine farrowing building, and an animal feed (corn) operation were used to generate aerosols in a $1 \mathrm{~m}^{3}$, Plexiglas laminar flow chamber as previously described. ${ }^{37}$ During each aerosol generation experiment, a total of 14 side-by-side samples were collected on $37 \mathrm{~mm}$ glass fiber filters (Gelman Sciences, Ann Arbor, MI), with cellulose support pads (Gelman Sciences, Ann Arbor, MI) at flow rates of approximately $21 \mathrm{~min}^{-1}$. As described previously, initial experiments to evaluate the homogeneity of generated aerosols found coefficients of variation for gravimetric analysis (coefficient of variation, $\mathrm{CV}=6 \%$ to $12 \%)$ and endotoxin assay $(13 \%-15 \%) .{ }^{37}$

A total of seven aerosol generation sessions, or experiments, were performed for each dust type. The sample times were varied from 10 to $50 \mathrm{~min}$ to produce a range of endotoxin loadings on the sample filters, therefore results are analyzed as concentrations per filter and not normalized to volume. After sample collection the backs of filter cassettes were attached to cassettes containing silica gel desiccant and stored at $4{ }^{\circ} \mathrm{C}$ until shipped in insulated boxes with freeze packs. Each laboratory received 14 randomly selected samples (two from each dust generation session) and one blank for each dust type, generating different dust and endotoxin levels. The extraction and assay methods used by each laboratory have been reported in detail previously. ${ }^{37}$ Three laboratories used the QCL-1000 endpoint assay, and three used the Kinetic-QCL method. To minimize variability due to the use of different LAL lots, a known and potentially major source of variability, a single lot of LAL for endpoint assays, and one similar lot for kinetic assays, were provided. Cambrex Inc. supplied endotoxin assay kits with standardized LAL from two comparable lots-one lot for Endpoint assays and one lot for Kinetic assays. The functional parameters (i.e. potency, linearity of response) of the LAL and LPS control standard endotoxin (CSE) were defined using both U.S. Reference Standard EC6 and the European reference standard BRP-2. Five laboratories provided lyophilized aliquots of solutions extracted from the filters for GC/MS determination of 3-hydroxy fatty acids (LPS).

\section{GC-MS analysis}

After each laboratory extracted the filters, a lyophilized portion of each extract was sent to the laboratory of one of the authors (LL, Sweden) for GC-MS analysis. Each lyophilized sample was resuspended in $1 \mathrm{ml}$ of $4 \mathrm{M}$ anhydrous methanolic $\mathrm{HCl}$ and incubated at $100{ }^{\circ} \mathrm{C}$ for $18 \mathrm{~h}$ to produce methylesters of the 3-OHFAs present in LPS in the samples. The 3-OHFAs of $10,12,14$, and 16 carbon chain lengths were then determined as methyl ester/trimethylsilyl derivatives using gas chromatography quadrupole mass-spectrometry as previously described ${ }^{39}$ Because of the possibility of interfering compounds in detection of 3 -OHFA $\mathrm{C} 10: 0,{ }^{42}$ we computed the total 3-OHFAs and percentages based only on $\mathrm{C} 12: 0, \mathrm{C} 14: 0$, and C16:0 3-OHFAs. LPS concentration was computed as the sum of nanomoles of individual 3OHFA with chain lengths 12-16 divided by 4 to account for the 4 molecules of 3OHFAs assumed per molecule of LPS. The concentration was adjusted according to the lyophylized volume and the extraction volume to calculate a total nanomoles per filter. Potency of dust collected on each filter was defined as the EU per filter divided by the nanomoles per filter.

\section{Statistical analysis}

Statistical analyses were performed using SAS version 8.1 (SAS Institute, Inc., Cary, NC). The PROC Univariate procedure was used to evaluate the distributions of data. Since data were log-normally distributed, they were $\log 10$-transformed before proceeding with analysis. Differences between laboratories and dust types for biological activity and LPS were evaluated using a mixed analysis of variance with a random effect for replicate experiment within dust type. Laboratory and dust were defined as fixed effects. Different models were evaluated, starting with a base model that assumed a single between and within run variance parameter, without interactions between dust and laboratory. Other models included those with different within or between variances for each dust type and for each laboratory or included interaction variables between dust and laboratory. The performance of other models was compared by changes in the Akaike Information Criterion (AIC) relative to the base model. The slope of the relationship between biological activity and LPS concentration was also evaluated using mixed analysis of variance with laboratory, dust and LPS concentration as fixed effects. Spearman rank correlations were calculated to examine the relationship between endotoxin activity from the LAL assay and the individual $\mathrm{C} 12$ : 0 , C14 : 0, C16 : 0 3OHFAs and the total LPS recovered from filters. 


\section{Results}

Table 1 shows the geometric mean endotoxin bioactivity obtained with the LAL assay (EU per filter), the LPS concentration obtained by GC-MS (nm LPS per filter), and the ratio of these two measures referred to as the potency (EU per nm LPS). Results are presented separately for each dust type and laboratory. Nanomoles of LPS was computed as the sum of nanomoles of individual 3OHFA with chain lengths $12-16$ divided by 4 for the number of 3OHFAs assumed per molecule of LPS. The results for EU were reported previously, although some data processing errors, which were discovered for these analyses were removed. ${ }^{37}$ These processing errors were minor and did not affect conclusions drawn in our earlier paper. As shown earlier, clear differences between laboratories seem present in obtained bioactivity. A similar heterogeneity between laboratories was observed for the LPS and potency data. Numbers of samples analyzed per laboratory differ because samples were damaged during transport or treatment in the different laboratories. Overall, the crude endotoxin results do suggest clear differences between laboratories in endotoxin levels found (EU per filter) of maximally a factor $2-3$ for the arithmetic mean. The LPS concentration varies as well between laboratories, and this crude analysis suggests that the differences are considerably more than found for the LAL assay. The differences between laboratories and dusts for the LPS results indicate that filter treatment and extraction procedures play an important role, since LPS was analyzed in one laboratory using the same procedure for all samples.

A more detailed analysis, for C12, C14 and C16 LPS chain lengths separately is presented in Table 2 . Here also, differences between dusts and laboratories could be observed. The high yields for laboratory B seem to be explained mainly by the contribution of C14 to LPS concentration. Table 2 shows the relative proportions of each of the three 3-OHFAs by laboratory and dust type and a mixed analysis of variance performed for each of the 3-OHFA, controlled for a random effect of experiment within dust type. The significant effects show that the proportion of each 3-OHFA in each extract assayed varied by dust type, by lab, and by dust type within lab. Examination of the least-square means (not shown) shows that only labs E and $\mathrm{F}(p=0.24)$ and labs $\mathrm{D}$ and $\mathrm{F}(p=0.07)$ obtained similar proportions of $\mathrm{C} 12$ : 0 . Only labs A and E obtained similar proportions of $\mathrm{C} 14: 0(p=0.09)$. Labs $\mathrm{B}$ and $\mathrm{D}(p=0.13)$ and labs B and $\mathrm{F}(p=0.47)$ obtained similar proportions of $\mathrm{C} 16$ : 0 . This indicates that even labs that were using very similar extraction methods (labs A, E, and F) often extracted a different fraction of the total LPS from the samples.

To explore these differences further, and correct for the effect of different endotoxin production experiments, a mixed analysis of variance was undertaken for each of these parameters to examine the effect of dust type and laboratory. For bioactivity (EU per filter), data were best described by a model, which contained terms to allow for different between and within dust variances (lowest AIC 346.6 versus 401.5 for the base model) and an interaction between dust type and laboratory. Chicken dust had a higher within than between run variance $(0.60$ versus 0.35 ), while swine dust showed a small within run variance compared to the between run variance $(0.18$ versus 0.46$)$. Corn dust showed a clearly lower within run variance compared to the between run variance $(0.05$ versus 0.29$)$. The within run variances observed for the biological activity measurements (EU) correspond with within run Geometric Standard Deviations (GSDs) of 2.2, 1.5 and 1.3, respectively for chicken, swine and corn dust. For the LPS measurements the variance component structure was somewhat different with within and between run variances of 0.5 and 0.26 for chicken dust, 0.55 and 0.31 for swine dust and 0.35 and 0.05 for corn dust. The within run variances correspond to GSDs of 2.0, 2.1 and 1.8 for chicken, swine and corn dust, respectively. Especially the GSDs for the biological activity (EU) of chicken dust and to a lesser extent swine dust are high and reflect considerable differences in yield for each sample within an experimental run. The reasons for these differences are not understood. The between run variability reflects how successful attempts were made to obtain aerosol generation of dust from the same environment with different endotoxin levels. Differences within a run are theoretically not desirable, and may reflect heterogeneity in loading of dust samples, for instance due to fluctuations in particle size distribution or endotoxin content of particulates.

The results of the mixed model are presented as estimated values of this model with confidence limits in Table 3. As

Table 1 Geometric mean and minimal and maximal endotoxin bioactivity (LAL-test), LPS concentration (GC-MS), and potency of aqueous filter extracts made in five laboratories

\begin{tabular}{|c|c|c|c|c|c|c|c|c|c|c|}
\hline \multirow[b]{2}{*}{ Laboratory } & \multicolumn{4}{|c|}{ Bioactivity (EU) per filter ${ }^{a}$} & \multicolumn{3}{|c|}{ LPS concentration per filter ${ }^{b}$} & \multicolumn{3}{|c|}{ EU per unit of LPS ${ }^{c}$} \\
\hline & $\mathrm{N}$ & GM & Min & Max & GM & Min & Max & GM & Min & Max \\
\hline \multicolumn{11}{|l|}{ Chicken } \\
\hline A & 14 & 321 & 204 & 687 & 0.069 & 0.024 & 0.130 & 4470 & 1620 & 12600 \\
\hline B & 12 & 388 & 110 & 2490 & 0.095 & 0.034 & 0.631 & 3310 & 2350 & 6770 \\
\hline D & 13 & 305 & 42 & 4656 & 0.025 & 0.012 & 0.107 & 12130 & 3580 & 47400 \\
\hline $\mathrm{E}$ & 13 & 539 & 176 & 2969 & 0.022 & 0.003 & 0.202 & 24550 & 9960 & 98600 \\
\hline $\mathrm{F}$ & 14 & 311 & 83 & 1683 & 0.020 & 0.005 & 0.079 & 15140 & 3980 & 24350 \\
\hline \multicolumn{11}{|l|}{ Swine } \\
\hline A & 14 & 112 & 46 & 392 & 0.364 & 0.101 & 1.350 & 310 & 70 & 1200 \\
\hline $\mathrm{B}$ & 14 & 97 & 34 & 404 & 1.994 & 0.421 & 8.140 & 50 & 4 & 170 \\
\hline D & 14 & 320 & 61 & 1350 & 0.127 & 0.023 & 0.462 & 2510 & 720 & 13900 \\
\hline $\mathrm{E}$ & 11 & 88 & 17 & 291 & 0.304 & 0.016 & 1.898 & 290 & 50 & 5190 \\
\hline $\mathrm{F}$ & 13 & 48 & 13 & 150 & 0.069 & 0.025 & 0.195 & 700 & 470 & 990 \\
\hline \multicolumn{11}{|l|}{ Corn } \\
\hline A & 13 & 159 & 72 & 278 & 0.020 & 0.006 & 0.226 & 8130 & 470 & 45500 \\
\hline B & 14 & 196 & 73 & 404 & 0.003 & 0.003 & 0.105 & 5890 & 3830 & 19370 \\
\hline $\mathrm{D}$ & 14 & 441 & 158 & 1168 & 0.024 & 0.014 & 0.039 & 18580 & 8340 & 35900 \\
\hline $\mathrm{E}$ & 13 & 57 & 23 & 173 & 0.016 & 0.007 & 0.041 & 3630 & 1050 & 7830 \\
\hline $\mathrm{F}$ & 12 & 104 & 77 & 291 & 0.010 & 0.004 & 0.024 & 10480 & 6510 & 20100 \\
\hline
\end{tabular}


Table 2 Percentage of 3-hydroxy fatty acids $(\mathrm{C} 12,14$, and 16) recovered from three types of dust samples by five laboratories descriptive data and mixed analysis of variance

\begin{tabular}{|c|c|c|c|c|c|c|c|c|c|}
\hline \multirow[b]{2}{*}{ Laboratory } & \multicolumn{3}{|c|}{ C12:0 } & \multicolumn{3}{|c|}{ C14:0 } & \multicolumn{3}{|c|}{ C16:0 } \\
\hline & $\%$ & Min & Max & $\%$ & Min & $\operatorname{Max}$ & $\%$ & Min & $\operatorname{Max}$ \\
\hline \multicolumn{10}{|l|}{ Chicken } \\
\hline A & 62 & 40 & 82 & 16 & 5 & 25 & 22 & 10 & 37 \\
\hline B & 13 & 0 & 19 & 43 & 12 & 51 & 44 & 33 & 73 \\
\hline $\mathrm{D}$ & 24 & 16 & 40 & 24 & 19 & 35 & 52 & 41 & 61 \\
\hline $\mathrm{E}$ & 19 & 11 & 24 & 22 & 11 & 36 & 59 & 45 & 73 \\
\hline $\mathrm{F}$ & 16 & 9 & 33 & 41 & 11 & 56 & 43 & 32 & 65 \\
\hline \multicolumn{10}{|l|}{ Swine } \\
\hline A & 74 & 17 & 91 & 8 & 2 & 36 & 18 & 6 & 80 \\
\hline B & 4 & 0 & 7 & 29 & 23 & 70 & 67 & 23 & 73 \\
\hline $\mathrm{D}$ & 9 & 5 & 25 & 21 & 16 & 53 & 70 & 22 & 77 \\
\hline $\mathrm{E}$ & 5 & 4 & 8 & 18 & 14 & 21 & 77 & 75 & 81 \\
\hline $\mathrm{F}$ & 9 & 7 & 13 & 32 & 23 & 40 & 59 & 50 & 68 \\
\hline \multicolumn{10}{|l|}{ Corn } \\
\hline A & 16 & 0 & 51 & 19 & 0 & 31 & 66 & 18 & 100 \\
\hline B & 11 & 0 & 23 & 39 & 20 & 49 & 50 & 40 & 80 \\
\hline $\mathrm{D}$ & 26 & 1 & 33 & 24 & 1 & 32 & 50 & 3 & 57 \\
\hline $\mathrm{E}$ & 17 & 2 & 31 & 10 & 0 & 17 & 73 & 59 & 98 \\
\hline $\mathrm{F}$ & 23 & 13 & 54 & 24 & 4 & 45 & 53 & 13 & 74 \\
\hline Dust & \multicolumn{3}{|c|}{$p=0.0018$} & \multicolumn{3}{|c|}{$p=0.0043$} & \multicolumn{3}{|c|}{$p<0.0001$} \\
\hline $\mathrm{Lab}$ & \multicolumn{3}{|c|}{$p<0.0001$} & \multicolumn{3}{|c|}{$p<0.0001$} & \multicolumn{3}{|c|}{$p<0.0001$} \\
\hline Lab*Dust & \multicolumn{3}{|c|}{$p<0.0001$} & \multicolumn{3}{|c|}{$p<0.0001$} & \multicolumn{3}{|c|}{$p<0.0001$} \\
\hline
\end{tabular}

shown, the different inter and intra dust variances lead to differences in confidence intervals for different dusts. The estimated geometric mean concentrations are very similar, but for all laboratories other than lab D, chicken dust had a significant higher bioactivity. Laboratory E yielded a lower bioactivity than all other labs. The predicted geometric means for LPS are also given in Table 3. Here similar between and within run variance components for each dust type lead to a model that was only marginally less optimal compared to a model with different between dust variance components, and so estimates from the simpler model with similar within and between variance components for each dust are being used Again, there were significant interactions between dust types and the laboratories. For all labs but D, chicken dust had the highest bioactivity. All laboratories had higher LPS in swine dust compared to other dusts. Laboratory B yielded the highest concentrations of LPS for swine dust, and chicken dust. Concentrations of LPS were similar for all laboratories for corn dust, but laboratory B had the highest bioactivity and $\mathrm{E}$ the lowest.

Table 4 and Fig. 1 show the results of an analysis of the relationship between biological activity and LPS concentration

Table 3 Adjusted geometric mean endotoxin bioactivity (EU) and LPS concentration (GC-MS) by laboratory and dust type obtained by mixed models assuming different within and between run variances for each dust for both endotoxin bioactivity and LPS concentration

\begin{tabular}{|c|c|c|c|c|c|c|}
\hline Laboratory & EU filter ${ }^{a}$ & $\mathrm{Lcl}^{b}$ & $\mathrm{Ucl}^{b}$ & $\mathrm{~nm}$ filter ${ }^{c}$ & Lcl & $\mathrm{Ucl}$ \\
\hline \multicolumn{7}{|l|}{ Chicken } \\
\hline A & $308^{d}$ & 155 & 613 & 0.068 & 0.037 & 0.128 \\
\hline B & $441^{d}$ & 218 & 888 & 0.105 & 0.056 & 0.197 \\
\hline $\mathrm{D}$ & 321 & 160 & 642 & 0.027 & 0.014 & 0.050 \\
\hline $\mathrm{E}$ & $532^{d}$ & 266 & 1065 & 0.021 & 0.012 & 0.040 \\
\hline $\mathrm{F}$ & $311^{d}$ & 156 & 618 & 0.020 & 0.011 & 0.038 \\
\hline \multicolumn{7}{|l|}{ Swine } \\
\hline A & 112 & 68 & 185 & 0.364 & 0.188 & 0.703 \\
\hline $\mathrm{B}$ & 98 & 59 & 162 & 1.997 & 1.034 & 3.856 \\
\hline $\mathrm{D}$ & $321^{e}$ & 193 & 530 & 0.127 & 0.066 & 0.245 \\
\hline E & 98 & 57 & 168 & 0.347 & 0.176 & 0.684 \\
\hline $\mathrm{F}$ & 48 & 29 & 80 & 0.072 & 0.037 & 0.139 \\
\hline \multicolumn{7}{|l|}{ Corn } \\
\hline A & 170 & 110 & 266 & 0.020 & 0.014 & 0.030 \\
\hline B & 195 & 125 & 304 & 0.033 & 0.023 & 0.048 \\
\hline $\mathrm{D}$ & 443 & 285 & 690 & 0.024 & 0.017 & 0.034 \\
\hline $\mathrm{E}$ & $55^{f}$ & 36 & 87 & 0.016 & 0.011 & 0.023 \\
\hline $\mathrm{F}$ & 112 & 71 & 174 & 0.010 & 0.007 & 0.015 \\
\hline
\end{tabular}


Table 4 Adjusted slope of endotoxin bioactivity (EU) regressed on LPS concentration (GC-MS) by laboratory and dust type obtained by mixed models with different within and between variance components for each dust separately

\begin{tabular}{lrrc}
\hline Laboratory & Slope & SE & $P$ \\
\hline Chicken & & & \\
A & 0.19 & 0.27 & 0.46 \\
B & 0.95 & 0.15 & $<0.0001$ \\
D & 1.78 & 0.16 & $<0.0001$ \\
E & 0.68 & 0.10 & $<0.0001$ \\
F & 0.88 & 0.13 & $<0.0001$ \\
& & & \\
Swine & & & \\
A & -0.29 & 0.25 & 0.26 \\
B & -0.57 & 0.24 & 0.02 \\
D & 0.26 & 0.22 & 0.24 \\
E & -0.08 & 0.18 & 0.65 \\
F & 0.59 & 0.34 & 0.09 \\
Corn & & & \\
A & & & 0.28 \\
B & -0.08 & 0.08 & 0.07 \\
D & 0.16 & 0.09 & 0.0005 \\
E & 0.82 & 0.22 & 0.03 \\
F & 0.32 & 0.14 & 0.41 \\
\hline
\end{tabular}

for each dust and laboratory. Clearly different slopes were observed for each laboratory and each dust. In all laboratories but laboratory A biological activity in chicken dust was positively associated to LPS concentration. The slope in lab D was steeper and differed significantly from laboratory B, E and F. For swine dust biological activity and LPS levels were only significantly associated in laboratory $\mathrm{B}$, but this was a
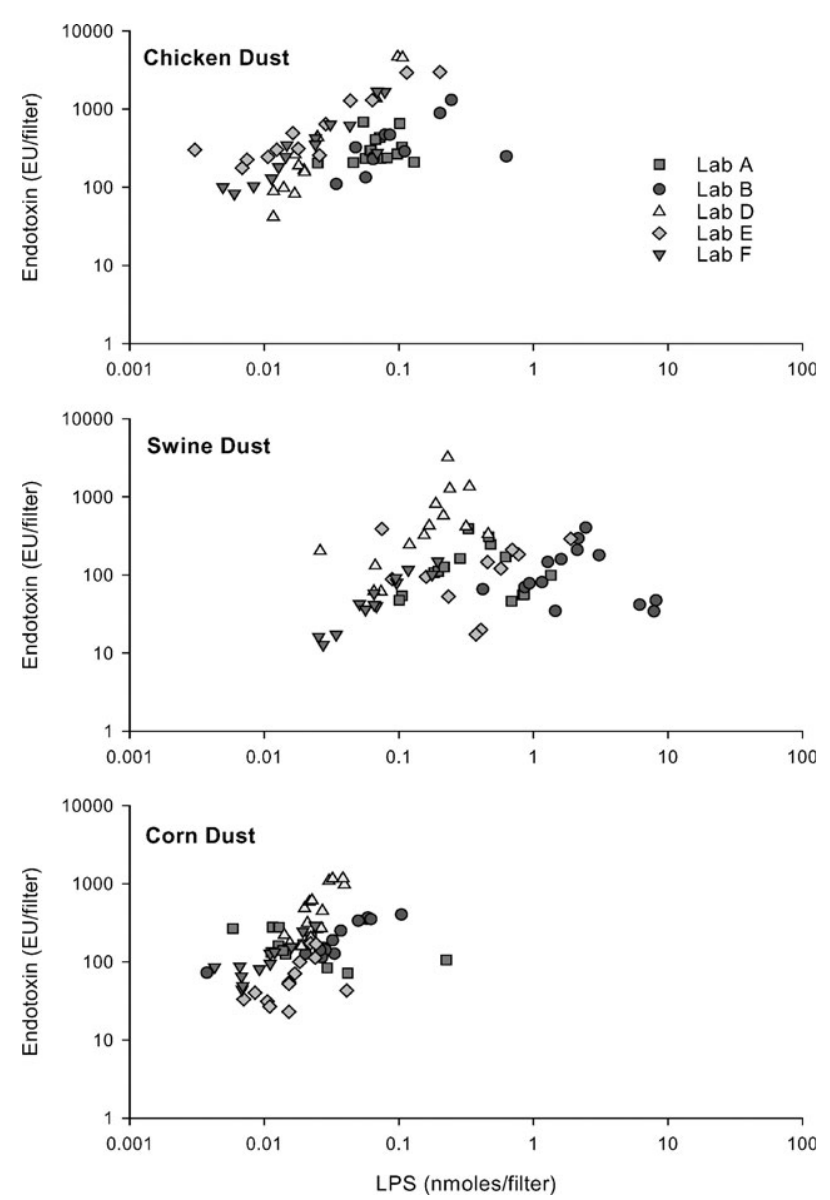

Fig. 1 LAL $v s$. LPS by laboratory and dust type. negative association. For corn dust biological activity was only positively associated to LPS concentration in laboratories D and E. The relatively narrow range of LPS recoveries and endotoxin activity measurements evident in the corn dust plot may account for the failure to find more clear cut associations in the parametric analysis of corn dust.

\section{Discussion}

We used GC-MS analysis of 3-OHFAs as a measure of LPS concentrations in aqueous filter extracts produced by five laboratories to determine whether differences in LPS extraction efficiency between laboratories could account for differences in endotoxin bioactivity detected by Limulus assay. The consistent interaction between laboratory and dust type for endotoxin activity indicates that comparisons of laboratory performance should take into account the environmental source of the endotoxin. The differences between laboratories were a factor of 2-3 maximally for the LAL assay, while differences for LPS were somewhat larger. Variability in extraction efficiency resulting from physical-chemical effects of the sample matrix may be the most likely explanation for this observation. It is clear that extraction plays an important role in the variability found between laboratories. Each lab extracted different proportions of 3OHFAs and the extraction efficiency seemed also dependent on chain length. The swine dust used in these experiments had consistently larger amounts of LPS and lower endotoxin activity than chicken or corn dust. Thus, differences in LPS content did not explain differences in endotoxin bioactivity measurements between dust types. The extracts made in each of the five participating labs contained significantly different proportions of each of the 3-OHFAs measured. Thus, the extracts made by the five laboratories differed qualitatively as well as quantitatively with regard to the LPS recovered.

The dusts themselves differed in the amount of LPS accessible to LAL assays, and they may be different toxicologically. The consistent interaction between laboratory and dust for LPS indicates that extraction procedures play a major role and contribute to differences between laboratories, because in this comparison the analysis of LPS was done by one lab, but the extracts were produced by different laboratories using different extraction procedures. The mechanisms underlying these differences are not well understood, and this requires further evaluation.

The fact that in some cases high within run variances were observed (after correction for laboratory and dust effects) suggests that the dust generation did not always lead to homogeneous samples. It is also noteworthy that differences in variability were found for different dusts, which might reflect different particle size distributions for the different dusts. The within and between run variances for LPS measurement are somewhat different compared to the biological activity (EU) which suggests that these measurement techniques measure different quantities. These results illustrate that production of homogeneous samples is an important issue that deserves greater attentions in this type of study and rigid quality control is needed.

Across all laboratories, endotoxin activity showed a weak but significant correlation with the amounts of the $\mathrm{C} 12: 0$ and C14 : 0, but not the C16 : 0 3-OHFAs. The tendency for correlations to be strongest with the shorter chain 3-OHFAs has been previously reported in analyses of house dust. ${ }^{28,42}$ However, within laboratory and dust type, we observed no tendency for short chain fatty acids to be more highly correlated with bioactivity. Because of concern about interference, ${ }^{42}$ C10 : 0 3-OHFA was not included in the calculations. Thus, future comparisons of bioactivity with 3-OHFAs determined by GC-MS-MS may improve on the results reported here. Zhiping et al. ${ }^{7}$ studied respiratory disease in workers exposed to swine dust and incorporated measurement of endotoxin 
activity and LPS using LAL and GC-MS assays, respectively. The two methods were moderately correlated $(r=0.50)$. By comparison, two of the labs in this study had stronger correlations and three had lower, non-significant or no correlation between bioactivity and LPS content of the swine dust aerosol. In Zhiping's study, exposures determined using both methods correlated weakly with increased serum interleukin -6 , with the LAL LPS showing the highest correlation. LPS determined by LAL also correlated with increased respiratory symptoms, increased bronchial responsiveness, and decreased vital capacity. This corroborates with the overall experience in this field that LAL results clearly correlate with respiratory, systemic symptoms, and spirometry results. LPS measurements have not been widely used in these type of studies and the usefulness has to be established.

This study more thoroughly evaluated relationships between mass spectrometric and LAL assay results for multiple laboratories measuring endotoxin in agricultural dusts. Two important sources of variability, filter type to capture dust and LAL reagents, were controlled for. Aerosolized samples were collected using glass fiber filters and all laboratories used one of two standardized lots of reagents and LPS standard. Participating laboratories stored, extracted, and assayed the samples using their usual procedures, which differed. Laboratories A, $\mathrm{C}, \mathrm{E}$, and $\mathrm{F}$ used sterile pyrogen-free water for extraction and dilutions, while laboratory $\mathrm{D}$ added a surfactant $(0.05 \%$ Tween 20) and laboratory B used a buffer ( 0.05 M KPO4, $0.01 \%$ triethylamine). Extraction volumes varied from 5 to 30 $\mathrm{ml}$, extraction containers were either borosilicate glass or polypropylene, and samples were rocked, rotated, or sonicated during extraction. Extractions were carried out at room temperature for all labs except $\mathrm{A}$ and $\mathrm{B}$, which used controlled temperatures of $22{ }^{\circ} \mathrm{C} \pm 2{ }^{\circ} \mathrm{C}$, and $20^{\circ} \mathrm{C} \pm 2{ }^{\circ} \mathrm{C}$, respectively. Laboratories D, E and F stored extractions at $-20^{\circ} \mathrm{C},-20^{\circ} \mathrm{C}$, and $-70{ }^{\circ} \mathrm{C}$, respectively until assays were performed. Unfortunately, we were not able to perform direct hydrolysis of representative filters from every experimental aerosol. Therefore, we cannot compute the yield of LPS via aqueous extraction in each of the labs.

The three labs (D, E, and F) that froze and thawed extracts prior to bioactivity measurement had more consistent correlations between endotoxin activity and LPS content than did the two labs (A and B) that assayed extracts immediately after extraction. This may suggest that freezing extracts before analysis, while implicated in variable bioactivity loss in previous reports, ${ }^{33,34}$ may also have beneficial effects on the Limulus assay.

Laboratory B was the only laboratory that did not centrifuge extracts to remove dust particles. The consistently higher amounts of LPS in the filter extracts from laboratory B compared with all other labs, therefore, likely represents particle-associated LPS that was systematically removed by centrifugation in the other laboratories. The relative similarity of the EU per filter determined by lab B and other labs suggests that although lab B assayed extracts containing larger amounts of particle associated LPS than other labs, the additional particle associated LPS did not react with the Limulus amebocyte lysate. This finding is consistent with results from human inhalation experiments with isolated and cell bound endotoxin ${ }^{43}$ and with older observations that LAL does not react with cell-bound LPS. ${ }^{44}$ If particle bound LPS dominated Lab B's extracts and Limulus reactive LPS was a small fraction and poorly correlated with total LPS in Lab B's extracts, that could account for the weak association between endotoxin activity and LPS in Lab B data. This also suggests that the three labs with the lowest LPS recoveries and the strongest endotoxin-LPS associations may have preferentially removed most of the cell-bound LPS through centrifugation and rendered the remainder more Limulus reactive through freezing their extracts before Limulus assay.
Potency $\left(\mathrm{EU} \mathrm{nm} \mathrm{nm}^{-1}\right.$ ) was found to vary depending on the type of dust and on the specific laboratory. Swine dust contained approximately an order of magnitude more LPS than chicken or corn dust, yet it was no more biologically active in the Limulus assay. As a result, the estimated potency for the LPS in swine dust was much lower than for chicken or corn dust. Factors that may influence the potency of a dust sample in the Limulus assay include differences in the proportion of cell or membrane bound LPS to free LPS as discussed above, as well as differences in chemical structure of LPS of the resident microbial flora. ${ }^{28,42}$ Dusts from swine barns contain fecal bacteria that would be expected to be highly active in the Limulus assay. Thus, we expected swine dust to be at least as potent as chicken barn dust and more potent per nanomole than corn dust. Given that we found the reverse, it seems likely that a large proportion of LPS in swine dust is cell bound and unavailable for reaction with LAL. Experimental data suggest that cell bound LPS may still be highly biologically active when inhaled. ${ }^{43}$ This indicates a need to determine whether the Limulus assay accurately reflects the potential human toxicity of endotoxin across different types of dust samples and environments.

\section{Conclusions}

There were significant differences in endotoxin assay and GCMS (LPS) results between laboratories for all three dust types. The pattern of differences between labs varied by dust type, and evaluation of assay versus GC-MS results also yielded different relationships between laboratories. Each laboratory extracted different amounts of 3-hydroxy fatty acids, however in general more LPS was recovered from swine dust. Overall, the differences in potency $\left(\mathrm{EU} \mathrm{nm}{ }^{-1}\right)$ between the three types of dust were highly significant. Particle-associated LPS is most likely removed by centrifugation, but when present was not detected by the LAL assay. In conclusion, extraction method is a significant factor contributing to differences between laboratories, that requires further evaluation. Standardization of extraction method is likely to be an important step in obtaining comparable performance among laboratories. New studies should include more dusts from different environments, and LPS analysis should be applied to extracts produced by different labs, but also to a complete set of parallel samples to be able to study the effect of extraction procedures more closely. In addition, production of homogeneous dust samples with small differences within test runs deserves more attention. A fractional factorial design with larger sample sizes may also be beneficial. This report demonstrates that both chemical and biological assay methods can be important tools in further understanding endotoxin exposure and its relationship to respiratory disease across a variety of environments. These data suggest that LAL methods may work best when comparing exposures within similar environments, and when the proportion of cell-free to cell-bound endotoxin is more or less constant. Mass spectrometry gives additional information which seems helpful in optimizing extraction and sample treatment procedures. GC-MS may have advantages when comparing exposures across heterogeneous environments and when the proportion of cell-free to cell-bound endotoxin varies from sample to sample. At present, however, there is little data relating mass spectrometry measurements of LPS with health effect. Future studies of endotoxin and human health should consider incorporating measurement of 3-OHFAs as well as Limulus assay.

\section{Acknowledgements}

The authors thank Marsha O'Neill and Jeanine DeKoster for technical assistance; Steve Olenchock and Robert Woolson for advice; and Ron Berzofsky and Cambrex (BioWhittaker) for providing standardized lots of LAL reagents and assay kits. 
This work was funded by the CDC/NIOSH University of Iowa Great Plains Center for Agricultural Health (NIOSHU07 CCU 706145-05), Environmental Health Sciences Research Center Grants (NIH-P30 ES05605 and P30 ES00002), NIEHS Grant R01 ES-07036, NIOSH Grant R01 OH03489, and the High Plains Intermountain Center for Agricultural Health (NIOSH - U50 OH008085). Jeroen Douwes is supported by a research fellowship from the Netherlands Organisation for Scientific Research (NWO).

\section{References}

1 R. Rylander, P. Haglind and M. Lundholm, Am. Rev. Respir. Dis. 1985, 131, 209-213.

2 R. M. Castellan, S. A. Olenchock, K. B. Kinsley and J. L. Hankinson, New Engl. J. Med., 1987, 317, 605-610.

3 T. Smid, D. Heederik, R. Houba and P. H. Quanjer, Am. J. Ind Med., 1994, 25, 877-888.

4 D. A. Schwartz, P. S. Thorne, S. J. Yagla, L. F. Burmeister, S. A. Olenchock and J. L. Watt et al., Am. J. Respir. Crit. Care Med. 1995, 152, 603-608.

5 D. K. Milton, D. Wypij, D. Kriebel, M. Walters, S. K. Hammond and J. Evans, Am. J. Ind. Med., 1996, 29, 3-13.

6 S. J. Reynolds, K. J. Donham, P. Whitten, J. A. Merchant, L. F. Burmeister and W. J. Popendorf, Am. J. Ind. Med., 1996, 29, 33-40.

7 W. Zhiping, P. Malmberg, B.-M. Larsson, K. Larsson, L. Larsson and A. Saraf, Am. J. Respir. Crit. Care Med., 1996, 154, 12611266.

8 K. J. Donham, D. Cumro, S. J. Reynolds and J. A. Merchant, J. Occup. Environ. Med., 2000, 42, 260-269.

9 P. F. Vogelzang, J. W. van der Gulden, H. Folgering, D. Heederik, M. J. Tielen and C. P. van Schayck, Chest, 2000, 117, 14881495.

10 F. Gyntelberg, P. Suadicani, J. W. Nielsen, P. Skov, O. Valbjørn and P. A. Nielsen et al., Indoor Air, 1994, 4, 223-238.

11 S. J. Reynolds, D. W. Black, S. S. Borin, G. Breuer, L. F. Burmeister and L. J. Fuortes et al., Appl. Occup. Environ. Hyg., 2001, 16, 1065-1077.

12 O. Michel, J. Kips, J. Duchateau, F. Vertongen, L. Robert and H. Collet et al., Am. J. Respir. Crit. Care Med., 1996, 154, 16411646.

13 M. C. Rizzo, C. K. Naspitz, E. Fernandez-Caldas, R. F. Lockey, I. Mimica and D. Sole, Pediatr. Allergy Immunol., 1997, 8 121-126.

14 U. Gehring, G. Bolte, M. Borte, W. Bischof, B. Fahlbusch and H. E. Wichmann et al., J. Allergy Clin. Immunol., 2001, 108, 847-854.

15 J.-H. Park, D. R. Gold, D. L. Spiegelman, H. A. Burge and D. K. Milton, Am. J. Respir. Crit. Care Med., 2001, 163, 322-328.

16 J. E. Gereda, D. Y. M. Leung, A. Thatayatikom, J. E. Streib, M R. Price and M. D. Klinnert et al., Lancet, 2000, 355, 1680-1683.

17 C. Braun-Fahrlander, J. Riedler, U. Herz, W. Eder, M. Waser and L. Grize et al., New Engl. J. Med., 2002, 347, 869-877.

18 A. H. Liu, J. Allergy Clin. Immunol., 2002, 109, 379-392.
19 P. F. J. Vogelzang, J. W. J. Vandergulden, H. Folgering, J. J. Kolk, D. Heederik and L. Preller et al., Am. J. Respir. Crit. Care Med., 1998, 157, 15-18.

20 A. Rask-Anderson, P. Malmberg and M. Lundholm, Br. J. Ind. Med., 1989, 46, 412-416.

21 E. Kateman, D. Heederik, T. M. Pal, M. Smeets, T. Smid and M. Spitteler, Scand. J. Work Environ. Health, 1990, 16, 428-433.

22 K. B. Teeuw, C. M. Vandenbroucke-Grauls and J. Verhoef, Arch. Intern. Med., 1994, 154, 2339-2345.

23 C. J. Hines, D. K. Milton, L. Larsson, M. R. Petersen, W. J. Fisk and M. J. Mendell, Indoor Air, 2000, 10, 2-12.

24 Health Council of the Netherlands, Dutch Expert Committee on Occupational Standards (DECOS), Endotoxins 1998/03WGD, Health Council of the Netherlands, Rijswijk, 1998.

25 D. K. Milton, in Bioaerosols Assessment and Control, ed. J. Macher, D. K. Milton, H. A. Burge and P. Morey, American Conference of Governmental Industrial Hygienists, Cincinnati, 1999, 23-1-14

26 D. C. Morrison, S. W. Vukajlovich, J. L. Ryan and J. Levin, Prog Clin. Biol. Res., 1987, 231, 55-73.

27 H. Takada, S. Kotani, S. Tanaka, T. Ogawa, I. Takahashi and M. Tsujimoto et al., Eur. J. Biochem., 1988, 175, 573-580.

28 A. Saraf, L. Larsson, H. Burge and D. Milton, Appl. Environ. Microbiol., 1997, 63, 2554-2559.

29 S. A. Olenchock, D. M. Lewis and J. C. Mull, Scand. J. Work Environ. Health, 1989, 15, 430-435.

30 D. K. Milton, R. J. Gere, H. A. Feldman and I. A. Greaves, Am. Ind. Hyg. Assoc. J., 1990, 51, 331-337.

31 A. Hollander, D. Heederik, P. Versloot and J. Douwes, Am. Ind. Hyg. Assoc. J., 1993, 54, 647-653.

32 S. Reynolds and D. K. Milton, Appl. Occup. Environ. Hyg., 1993, 8, 761-767.

33 J. Douwes, P. Versloot, A. Hollander and D. Heederik, Appl. Environ. Microbiol., 1995, 61, 1763-1769.

34 D. K. Milton, D. K. Johnson and J.-H. Park, Am. Ind. Hyg Assoc. J., 1997, 58, 861-867.

35 P. S. Thorne, S. J. Reynolds, D. K. Milton, X. Zhang, P. D. Bloebaum and P. Whitten et al., Am. Ind. Hyg. Assoc. J., 1997, 58, 792-799.

36 D. T. W. Chun, V. Chew, K. Bartlett, T. Gordon, R. R. Jacobs and B.-M. Larsson et al., Appl. Occup. Environ. Hyg., 2000, 15, $152-157$.

37 S. J. Reynolds, P. S. Thorne, K. J. Donham, E. A. Croteau, K. M Kelly and D. Lewis et al., Am. Ind. Hyg. Assoc. J., 2002, 63, 430-438.

38 A. Sonesson, L. Larsson, A. Schutz, L. Hagmar and T. Hallberg, Appl. Environ. Microbiol., 1990, 56, 1271-1278.

39 Z. Mielniczuk, E. Mielniczuk and L. Larsson, J. Microbiol. Methods, 1993, 17, 91-102.

40 M. Walters, D. K. Milton, L. Larsson and T. Ford, Appl. Environ. Microbiol., 1994, 60, 996-1005.

41 A. Saraf and L. Larsson, J. Mass Spectrom., 1996, 31, 389-396.

42 A. Saraf, J.-H. Park, D. K. Milton and L. Larsson, J. Environ. Monit., 1999, 1, 163-168.

43 R. Rylander, B. Bake, J. J. Fischer and I. M. Helander, Am. Rev Respir. Dis., 1989, 140, 981-986.

44 J. H. Jorgensen and R. F. Smith, Proc. Soc. Exp. Biol. Med., 1974, 146, 1024-1031. 